\title{
Cognitive, affective, and social factors maintaining paranoia in adolescents with mental health problems: A longitudinal study.
}

Jessica C. Bird ${ }^{a *}$, Felicity Waite ${ }^{\mathrm{a}}$, Eleanor Rowsell ${ }^{\mathrm{b}}$, Emma C. Fergusson $^{\mathrm{a}}$, Daniel Freeman ${ }^{\mathrm{a}}$

Submitted to Psychiatry Research

Date: $17^{\text {th }}$ February 2017

\begin{abstract}
Paranoia may be a significant concern during adolescence, but there has been little research on excessive mistrust in young people. In this longitudinal study we set out to test the predictive ability of a number of cognitive, affective, and social factors in the early development of paranoia in a clinical adolescent population. Thirty four help-seeking adolescents, aged 11-16 years, reporting paranoid thoughts and attending mental health services were recruited. Self-report and interview assessments of paranoia were conducted at baseline. Measures relating to a cognitive model of persecutory delusions were completed. Paranoia was reassessed after three months with thirty three participants. Significant predictors of paranoia persistence were anxiety, depression, worry, negative self-beliefs, perceptual anomalies, insomnia, affective reactivity, bullying, and cyber victimization. No effect was found for reasoning bias or negative perceptions of academic ability, social competence, and physical appearance. In conclusion, many of the maintenance factors implicated in adult paranoia are likely to prove important in the early development of paranoia in young people. Further experimental and treatment studies are now needed to examine the causal role of these factors in the occurrence of paranoia in adolescents.
\end{abstract}

Keywords:

Psychosis; Delusions; Early-intervention; Child; Anxiety; Depression 


\section{Introduction}

Paranoia, the unfounded fear that others are intentionally trying to harm you (Freeman, 2016), may be a particular concern during adolescence. At this age rates of non-clinical paranoia are especially high (Freeman et al., 2011; Wigman et al., 2011) and such feelings of persecution are associated with anxiety, depression, low self-esteem, and conduct problems (Ronald et al., 2014; Wong et al., 2014). Furthermore, excessive mistrust during this sensitive developmental period may influence an adolescent's emerging social relationships, selfidentity, and successful transition into adulthood. Paranoia represents a distinct clinical entity existing on a continuum in youth (Ronald et al., 2014), ranging from mild suspiciousness and mistrust to persecutory delusions at the extreme (Freeman, 2016). This spectrum of experiences share an underlying aetiology, supported by evidence of a consistent heritability between mild and severe paranoia (Zavos et al., 2014). With the emergence of psychosis commonly occurring in youth (Kessler et al., 2007), persistent paranoid ideation at this age will likely precede the onset of delusions. Understanding the causal mechanisms in the early progression of paranoia may therefore prove important for early intervention.

In the past fifteen years there has been a significant advance in the understanding of paranoia in adults (Freeman, 2016). It is well established that the onset of persecutory delusions is influenced by a range of biopsychosocial risk factors theorised to increase an individual's sensitivity and affective reactivity to stressful life events (Freeman et al., 2002; Gibson et al., 2014; Palmier-Claus et al., 2012). However, paranoid ideation is common within the general population (Bebbington et al., 2013), leading to our view that paranoia is rooted within adaptive concerns about trust, vulnerability, and social evaluation. Within this framework we conceptualise persecutory delusions as unfounded threat beliefs, maintained by a range of cognitive and affective factors including low self-esteem, worry, sleep dysfunction, perceptual anomalies, and reasoning biases (Freeman, 2016).

Arguably no age is more characterised by perceived social vulnerability and threat than adolescence. Heightened social processing and catastrophic appraisal of perceived rejection follows from the social reorientation away from family and towards peer acceptance, and the neurodevelopmental changes in social cognition and affect regulation (Blakemore and Mills, 2014; Nelson et al., 2016; Sebastian et al., 2010). The stressful social world of adolescence, including online media, also provides a constant source of social comparison, ambiguous information, and opportunity for criticism and victimization (Best et al., 2014; Volk et al., 2006). Consequently, adolescence may provide the psychological conditions on which 
unfounded attributions of threat from others may flourish.

Very few studies have specifically examined paranoia in adolescents. However, a small number of cross-sectional studies with non-clinical youth show associations between paranoia and anxiety, depression, low self-esteem, insomnia, and hallucinatory experiences (Galbraith et al., 2014, Raes and van Gucht, 2009; Ronald et al., 2014; Taylor et al., 2015; Wong et al., 2014). For example, in a large study across the UK and Hong Kong, Wong et al. (2014) found children with high mistrust had significantly more internalising and externalising problems than peers with low mistrust. In the few clinical studies with adolescents, paranoia has also been associated with the presence and severity of social anxiety disorder (Pisano et al., 2015), negative life events, and emotional problems, although not reasoning biases (Ruffell et al., 2016).

The aim of this longitudinal study was to test if cognitive, affective, and social factors established in adult research (Freeman, 2016; Freeman et al., 2002) also contribute to the early development of persecutory ideas in help-seeking adolescents. Our first hypothesis was that key cognitive processes outlined by Freeman (2016) including negative affect, worry, negative self-beliefs, perceptual anomalies, sleep dysfunction, and reasoning bias (jumping to conclusions) would predict the persistence of paranoia. Negative self-beliefs was expanded to include core beliefs, self-esteem, and perceived academic ability, social competence, and physical appearance. Second, based on a stress-vulnerability framework of paranoia (Freeman et al., 2002), we hypothesised that heightened affective reactivity to stress (i.e. perceived stress and social media affective experience) and negative social experiences (i.e. peer bullying and cyber victimization) would also predict the persistence of paranoia in young people.

\section{Design and methods}

This was a longitudinal observational study. Participants completed a baseline assessment in which paranoia and predictor variables were measured, and after three months paranoia was reassessed.

\subsection{Participants}

A clinical sample of adolescents aged 11-16 years were recruited from specialist child and adolescent mental health services (CAMHS) within Oxford Health NHS Foundation Trust. Clinicians were approached to identify patients with suspected paranoid ideation, irrespective of psychiatric diagnosis. Thirty eight adolescents agreed to be contacted. Eligibility was established by self-reported paranoia, using the Green et al. Paranoid Thoughts Scale (GPTS; 
Green et al., 2008) and validation in a brief clinical interview. A GPTS inclusion threshold of 26 was set, based on data that less than $20 \%$ of the general adult population have this level of paranoia (Freeman et al., 2014). Participants were excluded if they scored below this threshold $(n=2)$ or showed no evidence of unfounded paranoia in the interview (e.g. reporting genuine hostility; $n=2$ ). Further exclusion criteria were an established diagnosis of Autism Spectrum Disorder, moderate or severe learning disability, or inability to complete questionnaires in English. Thirty four eligible participants were recruited. Informed parental consent and child assent was obtained prior to participation and all young people were reimbursed for their time. Full ethical approval from the Health Research Authority was obtained.

\subsection{Measures}

\subsubsection{Paranoia}

The Green et al. Paranoid Thoughts Scale (GPTS; Green et al., 2008) Part B was the primary outcome in this study. The GPTS includes 16 self-report items assessing persecutory thoughts over the past month. Each item is rated on a 1-5 scale ( $1=$ not at all, $5=$ totally $)$ with higher scores indicating greater paranoia. The scale has high test-retest reliability (intra-class correlation $=0.87)$ and internal consistency in adults with persecutory delusions $(\alpha=0.90)$ and has been used in adolescents with psychosis (Korver-Nieberg et al., 2013). In the baseline assessment the suspiciousness/persecution item of the Positive and Negative Syndrome Scale (PANSS; Kay et al., 1987) was used to clinically validate the presence of paranoia. The PANSS is an interview rating scale assessing the severity of schizophrenia symptoms over the past week. Symptoms are scored on a 1-7 scale $(1=$ absent, $7=$ extreme $)$ where higher scores indicate increased severity. The suspiciousness/persecution item has adequate internal consistency ( $\alpha=0.69$; Peralta and Cuesta, 1994).

\subsubsection{Affective symptoms}

The Revised Children's Anxiety and Depression Scale (RCADS; Chorpita et al., 2000) is a 47-item, self-report questionnaire of affective symptoms over the last month. The depression subscale and the total anxiety score (consisting of separation anxiety, social phobia, generalized anxiety disorder, panic disorder, and obsessive compulsive disorder) were used for this study. Items are scored on a 0-3 scale ( $0=$ never, $3=$ always), with higher scores indicating greater symptoms. The RCADS demonstrates good internal consistency ( $\alpha=0.78-0.88$ ) in clinical samples of youth (Chorpita et al., 2005). 


\subsubsection{Worry}

The Penn State Worry Questionnaire for Children (PSWQ-C; Chorpita et al., 1997) is a 14-item measure of trait worry. Items are rated on a 0-3 scale ( $0=$ never, $3=$ always $)$ with higher scores indicating greater worry. The PSWQ-C has good internal consistency $(\alpha=0.89)$ in clinical samples of youth (Pestle et al., 2008).

\subsubsection{Negative self-beliefs}

The 6-item negative-self subscale of the Brief Core Schema Scales (BCSS; Fowler et al., 2006) was used as a measure of negative core self-beliefs. Each item is scored on a 0-4 scale ( 0 = not at all, $4=$ totally) with higher scores indicating greater negative self-beliefs. The subscale has good internal consistency $(\alpha=0.84$ ) and has been used in adolescents in CAMHS (Noone et al., 2015). The 10-item Rosenberg Self-Esteem Scale (RSES; Rosenberg, 1965) was used as a self-report measure of global self-esteem. Items refer to general feelings about the self and are rated on a 0-4 scale ( 0 = strongly disagree, $3=$ strongly agree), with lower scores indicating lower self-esteem. The RSES has good internal consistency ( $\alpha=0.86$; Sinclair et al., 2010) and has been used in adolescents with paranoia (Raes and Van Gucht, 2009). Finally, the social competence, academic competence, and physical appearance subscales of the SelfPerception Profile (SPP; Harter, 2012) were used to measure adolescent self-concepts. Each subscale consists of five items where participants choose whether they are most like one of two polarised descriptions using "sort of true" or "really true”. Items are scored on a 0-3 scale where higher scores reflect more positive self-judgements. The scales display good internal consistency ( $\alpha=0.78-0.92)$ with adolescents.

\subsubsection{Perceptual anomalies}

The 9-item hallucinations subscale of the Specific Psychotic Experiences Questionnaire (SPEQ; Ronald et al., 2014) was used to measure how frequently participants experience a range of perceptual anomalies. Each item is rated on a 0-5 scale ( $0=$ not at all, $5=$ daily) with higher scores indicating greater perceptual anomalies. The authors report good internal consistency ( $\alpha=0.87$ ) with adolescents.

\subsubsection{Insomnia}

Insomnia Severity Index (ISI; Bastien et al., 2001) is a 7-item self-report questionnaire assessing the severity of insomnia over the past two weeks. Items use a 5-point scale ( $0=n o t$ at all, $4=$ very much). Chung et al. (2011) demonstrate good internal consistency $(\alpha=0.83)$ in youth where the optimal total score for detecting clinical insomnia was 9 or above. 


\subsubsection{Jumping to conclusions}

Jumping to conclusions (JTC) was assessed with the 'beads task' (Garety et al., 2005), an established task of probabilistic reasoning. Participants are shown two jars of beads; one with 60 purple and 40 white beads and one with 40 purple and 60 white beads. Participants request as many beads from an unknown jar before deciding which one the beads are being taken from. The number of beads requested is the outcome, with a JTC bias determined by two or fewer beads (Garety et al., 2005).

\subsubsection{Affective reactivity}

The Perceived Stress Scale (PSS; Cohen et al., 1983) is a 14-item measure of the degree to which events are perceived to be over unpredictable, uncontrollable, and overloading during the past month. Each item is scored on a $0-4$ scale $(0=$ never, $4=$ very often $)$ with higher scores indicating higher perceived stress. The PSS has good internal consistency ( $\alpha=0.86)$ in clinical samples of adolescents (Martin et al., 1995). The negative affect subscale of the Social Networking Sites (SNSs) Usage Questionnaire (Shi et al., 2014) was used to measure affective reactivity to SNSs. Participants rate the frequency of experiencing four negative emotions whilst using SNSs. The 13-item usage subscale of the SNSs Usage Questionnaire was also administered to assess the frequency and duration of SNSs use. All items are rated using a 07 scale where higher scores indicate higher usage or frequency of emotions. The measure has been validated with young adults with good internal consistency $(\alpha=0.82)$.

\subsubsection{Negative social experiences}

The victim subscale of the Cyber Victim and Bullying Scale (CVBS; Çetin et al., 2011) is a 22-item self-report scale assessing the frequency of online cyber victimization. Participants rate each item on 1-5 scales ( 1 = never, 5 = always) with higher scores indicating greater cyber victimization. The CVBS has good internal consistency $(\alpha=0.89)$ in adolescents. The 16 -item Multidimensional Peer Victimization Scale (MPVS; Mynard and Joseph, 2000) was used as a self-report measure of peer bullying. Participants rate the frequency of each experience in the past year on a 0-3 scale ( $0=$ not at all, $1=$ once, 2 = more than once $)$, with higher scores indicating greater bullying. The scale has adequate internal consistency ( $\alpha=0.73-0.85$ ).

\subsection{Statistical analysis}

Analyses were conducted with SPSS for Windows (Version 24, SPSS, 2016). Rates of missing data were extremely low and most variables met criteria for parametric analyses. The key hypotheses were tested using Pearson's $r$ correlations of each predictive factor with follow-up 
paranoia as the dependant variable. These correlations were then repeated whilst co-varying for baseline paranoia severity. Non-parametric correlations were used for the CVBS and SPP social competence due to significantly non-normal distributions $(p<0.05)$. Two-tailed significance tests were used with the significance level unaltered at $\alpha=0.05$, agreeing with the view that "simply describing what [tests of significance] was done and why, and discussing the possible interpretations of each result" is the best approach for multiple comparisons (Perneger, 1998, p. 1236). We tested each variable separately, without using multiple regressions (see Miller and Chapman, 2001). Due to the relatively small sample the effect size was emphasised over significance and predictors with at least medium effect size $(r \geq 0.3$; Cohen, 1992) were highlighted. An a priori power analysis indicated that at least 30 participants would be needed to detect a medium effect size $(r \leq 0.3)$ with $80 \%$ power ( $p<$ 0.05).

\section{Results}

\subsection{Participant characteristics}

Thirty four participants with a mean age of $14.9(\mathrm{SD}=1.25)$ were recruited (Table 1). The sample was predominantly female and White British. Baseline GPTS scores did not differ according to participant gender $(t(32)=0.006, p=1.0)$ or medication status $(t(32)=1.54, p=$ 0.14). Most participants were supported by community CAMHS with only one participant seen by Early Intervention Services in Psychosis and two participants receiving inpatient care.

\subsection{Descriptive statistics}

\subsubsection{Baseline}

The descriptive statistics are shown in Table 2. The severity of self-report paranoia was high with mean scores comparable to adults with persecutory delusions (55.4 +/- 15.7; Green et al., 2008). Using the PANSS, participants' paranoid ideas were rated as Mild $(n=6)$, Moderate $(n=16)$, Moderate Severe $(n=6)$, or Severe $(n=4)$. High levels of depression, anxiety, worry, and negative self-beliefs were also reported. On the RCADS 73.5\% and 53\% participants scored above the clinical thresholds for depression and total anxiety, respectively. On the ISI, $88.2 \%$ of participants scored in the clinical range for insomnia. The data distribution for the physical appearance subscale of the SPP was significantly positively skewed $(z=2.97, p<0.01)$ where $50 \%$ of participants gave the lowest possible score. This 
variable was therefore excluded from the analysis. Only one participant displayed a JTC bias and therefore this variable also could not be included in the analysis.

\subsubsection{Follow up}

Follow up data was obtained for 33 of the original sample (97\%). The missing participant did not respond after being contacted. The mean GPTS score significantly reduced over the three months $(t(32)=2.65, p=0.01)$. The Reliable Change Index (RCI; Jacobson and Truax, 1991) examined individual GPTS scores where $11+$ points indicated a reliable change. A reliable reduction in GPTS score was observed for 12 participants (36\%) and four participants reported a reliable increase (12\%). Most participants were still in services at follow up with only four having been discharged. Eleven participants reported noteworthy treatment changes including a new psychological therapy $(n=4)$ or medication $(n=7)$. However, there was no difference in paranoia change over time between the treatment change group and remaining participants $(F(1,31)=0.78, p=0.39)$.

\subsection{Correlational analysis}

In line with the first hypothesis, negative affect, worry, negative views of the self (core beliefs and low self-esteem), perceptual anomalies, and sleep dysfunction significantly predicted paranoia severity after three months with medium to large effect sizes (see Table 3). Furthermore, worry ( $r=0.34, p=0.057$ ), negative core beliefs ( $r=0.34, r=0.059$ ), and insomnia ( $r=0.38, p=0.035$ ) continued to predict paranoia persistence with medium effect sizes after initial paranoia severity was adjusted, although only insomnia remained statistically significant. Although affective symptoms were highly correlated with paranoia severity, no independent effect was shown for anxiety or depression when baseline paranoia was adjusted. Contrary to the hypothesis, specific elements of negative self-perceptions including academic competence and social competence did not demonstrate associations with paranoia. Furthermore, there was no evidence of a jumping to conclusions reasoning bias in this sample.

In support of the second hypothesis, heightened perceived stress $(r=0.65, p<0.001)$, negative affect on social media $(r=0.53, p=0.002)$, peer bullying $(r=0.59, p<0.001)$, and cyber victimization $(r=0.39 p<0.001)$ significantly predicted paranoia persistence after three months with medium to large effect sizes. Medium effect sizes were maintained for all four variables after controlling for initial paranoia severity, although only perceived stress $(r=0.47$, $p=0.007)$ and negative affect on social media $(r=0.45, p=0.014)$ maintained statistical significance. 


\section{Discussion}

This is the first longitudinal study to examine the predictors of paranoia persistence specifically in adolescents with mental health problems. Despite very few participants seeking treatment for psychotic experiences, the severity of paranoia was high, with scores similar to patients with persecutory delusions (Green et al., 2008). The findings support the primary hypotheses, derived from a cognitive model of paranoia, that affective processes (anxiety, depression, worry, and negative self-schemas/low self-esteem), perceptual anomalies, and insomnia significantly predict paranoia persistence. Contrary to the hypothesis, no effect was observed for the jumping to conclusions reasoning bias or issues concerning adolescent selfconcepts (academic/social competence, physical appearance). Overall, many of the maintenance factors implicated in adult paranoia are likely to prove important in the early development of persecutory thinking in young people.

Psychosocial vulnerability factors were also found to predict the continuation of paranoia, specifically affective reactivity (i.e. heightened perceived stress and frequent negative affect when using social media) and negative social experiences (i.e. cyber victimization and peer bullying). Notably, perceived stress and negative affect on social media showed the strongest longitudinal associations with paranoia. In line with findings from the adult literature (Gibson et al., 2014; Palmier-Claus et al., 2012), these findings suggest heightened affective reactivity to social stress may play an important role in the emergence of paranoia during adolescence. Compared to adults, adolescents typically display increased emotion reactivity to perceived rejection and heightened neural processing of facial expressions and social emotions such as guilt (Blakemore, 2008; Goddings et al., 2012; Sebastian et al., 2010). This developmental hypersensitivity towards social processing, emotional reactivity, and overvaluation of peer acceptance may consequently provide the psychological conditions for the emergence of paranoia during this vulnerable age.

There are a number of clear limitations to this study. Most importantly, the sample is relatively small and the follow up length is short, both of which may reduce the ability to detect the associations with putative maintenance factors. Furthermore, the observational design cannot determine causality, with a potential for confounding in the observed correlations. With this in mind, a shared genetic propensity between paranoia and the predictors is possible (Shakoor et al., 2014). Another limitation was the potential for selection bias with participants 
identified by clinicians based on likely paranoia. However, the findings may have notable clinical implications. First, the high severity of paranoia in this sample of adolescents primarily with affective disorders suggests the significance of paranoia in youth extends beyond early intervention in psychosis services. Assessment and screening of paranoia in adolescent mental health services may therefore be important for effective early intervention. Second, with the majority of participants reporting persistent persecutory ideas the identification of clear maintenance factors provides potential avenues for treatment development. This is in line with adult research where psychological interventions targeting factors such as worry, negative selfbeliefs, and insomnia have been shown to indirectly reduce paranoia (Freeman et al., 2016, 2015a, 2015b, 2014). However, larger prospective studies using representative incidence samples from adolescent services with a longer follow up period are needed to strengthen the findings. Clinical intervention studies with young people targeting key maintenance factors can subsequently test the effect on persecutory thoughts. This will inform both the theory and treatment of paranoia in adolescents.

\section{Acknowledgements}

JB is currently supported by a National Institute of Health Research (NIHR) doctoral position. FW is an NIHR Fellow. DF is supported by an NIHR Research Professorship (Grant Number: RP-2014-05-003).

\section{Funding}

This research did not receive any specific grant from funding agencies in the public, commercial, or not-for-profit sectors.

\section{Conflict of Interest}

None. 


\section{References}

Bastien, C.H., Vallières, A., Morin, C.M., 2001. Validation of the insomnia severity index as an outcome measure for insomnia research. Sleep Med. 2, 297-307.

Bebbington, P.E., McBride, O., Steel, C., Kuipers, E., Radovanovic, M., Brugha, T., Jenkins, R., Meltzer, H.I., Freeman, D., 2013. The structure of paranoia in the general population. Br. J. Psychiatry, 202, 419-427. doi:10.1192/bjp.bp.112.119032

Best, P., Manktelow, R., Taylor, B., 2014. Online communication, social media and adolescent wellbeing: A systematic narrative review. Child. Youth Serv. Rev. 41, 27-36. doi:10.1016/j.childyouth.2014.03.001

Blakemore, S.-J., 2008. The social brain in adolescence. Nat. Rev. Neurosci. 9, 267-277. doi:10.1038/nrn2353

Blakemore, S.-J., Mills, K.L., 2014. Is adolescence a sensitive period for sociocultural processing? Annu. Rev. Psychol. 65, 187-207. doi:10.1146/annurev-psych-010213115202

Çetin, B., Yaman, E., Peker, A., 2011. Cyber victim and bullying scale: A study of validity and reliability. Comput. Educ. 57, 2261-2271. doi:10.1016/j.compedu.2011.06.014

Chorpita, B.F., Moffitt, C.E., Gray, J., 2005. Psychometric properties of the revised child anxiety and depression scale in a clinical sample. Behav. Res. Ther. 43, 309-322. doi:10.1016/j.brat.2004.02.004

Chorpita, B.F., Tracey, S.A., Brown, T.A., Collica, T.J., Barlow, D.H., 1997. Assessment of worry in children and adolescents: An adaptation of the penn state worry questionnaire. Behav. Res. Ther. 35, 569-581. doi:10.1016/S0005-7967(96)00116-7

Chorpita, B.F., Yim, L., Moffitt, C., Umemoto, L.A., Francis, S.E., 2000. Assessment of symptoms of DSM-IV anxiety and depression in children: A revised child anxiety and depression scale. Behav. Res. Ther. 38, 835-855. doi:10.1016/S0005-7967(99)00130-8

Chung, K.F., Kan, K.K.K., Yeung, W.F., 2011. Assessing insomnia in adolescents: Comparison of insomnia severity index, athens insomnia scale and sleep quality index. Sleep Med. 12, 463-470. doi:10.1016/j.sleep.2010.09.019

Cohen, J., 1992. A power primer. Psychol. Bull. 112, 155-159. doi:10.1037/00332909.112.1.155

Cohen, S., Kamarck, T., Mermelstein, R., 1983. A global measure of perceived stress. J. Health Soc. Behav., 24, 385-396. doi:10.2307/2136404 
Fowler, D., Freeman, D., Smith, B., Kuipers, E., Bebbington, P., Bashforth, H., Coker, S., Hodgekins, J., Gracie, A., Dunn, G., Garety, P., 2006. The brief core schema scales (BCSS): Psychometric properties and associations with paranoia and grandiosity in nonclinical and psychosis samples. Psychol. Med. 36, 749-59. doi:10.1017/S0033291706007355

Freeman, D., 2016. Persecutory delusions: A cognitive perspective on understanding and treatment. The Lancet Psychiatry 3, 685-692. doi:10.1016/S2215-0366(16)00066-3

Freeman, D., Bradley, J., Waite, F., Sheaves, B., Deweever, N., Bourke, E., ... Dunn, G., 2016. Targeting recovery in persistent persecutory delusions: A proof of principle study of a new translational psychological treatment (the Feeling Safe Programme). Behav. Cogn. Psychother. 44, 1-14. doi:10.1017/S1352465816000060

Freeman, D., Dunn, G., Startup, H., Pugh, K., Cordwell, J., Mander, H., et al, 2015a. Effects of cognitive behaviour therapy for worry on persecutory delusions in patients with psychosis (WIT): A parallel, single-blind, randomised controlled trial with a mediation analysis. Lancet Psychiatry. 2, 305-13. doi:10.1016/S2215-0366(15)00039-5

Freeman, D., Garety, P. a, Kuipers, E., Fowler, D., Bebbington, P.E., 2002. A cognitive model of persecutory delusions. Br. J. Clin. Psychol. 41, 331-347. doi:10.1348/014466502760387461

Freeman, D., McManus, S., Brugha, T., Meltzer, H., Jenkins, R., Bebbington, P., 2011. Concomitants of paranoia in the general population. Psychol. Med. 41, 923-936. doi:10.1017/S0033291710001546

Freeman, D., Pugh, K., Dunn, G., Evans, N., Sheaves, B., Waite, F., et al., 2014. An early Phase II randomized controlled trial testing the effect on persecutory delusions of using CBT to reduce negative cognitions about the self. Schizophr Res., 160, 186 - 192. doi:10.1016/j.schres.2014.10.038.

Freeman, D., Waite, F., Startup, H., Myers, E., Lister, E., McInerney, J., et al., 2015. Efficacy of cognitive behavioural therapy for sleep improvement in patients with persistent delusions and hallucinations (BEST): A prospective, assessor-blind, randomised controlled pilot study. The Lancet Psychiatry 2, 975 - 83. doi:10.1016/S2215-0366(15)00314-4

Galbraith, N.D., Manktelow, K.I., Chen-Wilson, C.-H., Harris, R.A., Nevill, A., 2014. Different combinations of perceptual, emotional, and cognitive factors predict three 
different types of delusional ideation during adolescence. J. Nerv. Ment. Dis. 202, 66876. doi:10.1097/NMD.0000000000000179

Garety, P.A., Freeman, D., Jolley, S., Dunn, G., Bebbington, P.E., Fowler, D.G., Kuipers, E., Dudley, R., 2005. Reasoning, emotions, and delusional conviction in psychosis. J Abnorm. Psychol. 114, 373-384. doi:10.1037/0021-843X.114.3.373

Gibson, L.E., Anglin, D.M., Klugman, J.T., Reeves, L.E., Fineberg, A.M., Maxwell, S.D., Kerns, C.M., Ellman, L.M., 2014. Stress sensitivity mediates the relationship between traumatic life events and attenuated positive psychotic symptoms differentially by gender in a college population sample. J. Psychiatry Res. 53, 111-118. doi:10.1016/j.jpsychires.2014.02.020

Goddings, A.L., Burnett-Heyes, S., Bird, G., Viner, R.M., Blakemore, S.J., 2012. The relationship between puberty and social emotion processing. Dev. Sci. 15, 801-811. doi:10.1111/j.1467-7687.2012.01174.x

Green, C.E., Freeman, D., Kuipers, E., Bebbington, P., Fowler, D., Dunn, G., Garety, P., 2008. Measuring ideas of persecution and social reference: The Green et al. Paranoid Thought Scales (GPTS). Psychol. Med. 38, 101-11. doi:10.1017/S0033291707001638

Harter, S., 2012. Self-perception profile for adolescents: Manual and questionnaires (revised). University of Denver, Denver.

Jacobson, N., Truax, P., 1991. Clinical significance: A statistical approach to defining meaningful change in psychotherapy research. J. Consult. Clin. Psychol., 59, 1219. doi:10.1037/0022-006X.59.1.12

Kay, S., Fiszbein, A., Opler, L., 1987. The positive and negative syndrome scale for schizophrenia. Schizophr Bull. 13, 261-276. doi:10.1093/schbul/13.2.261

Kessler, R.C., Amminger, G.P., Aguilar-Gaxiola, S., Alonso, J., Lee, S., Ustün, T.B., 2007. Age of onset of mental disorders: a review of recent literature. Curr. Opin. Psychiatry 20, 359-64. doi:10.1097/YCO.0b013e32816ebc8c

Korver-Nieberg, N., Fett, A., Meijer, C.J., Koeter, M., Shergill, S., de Haan, L., Krabbendam, L., 2013. Theory of mind, insecure attachment and paranoia in adolescents with early psychosis and healthy controls. Aust. N. Z. J. Psychiatry 47, 737-45. doi:10.1177/0004867413484370

Martin, R.A., Kazarian, S.S., Breiter, H.J., 1995. Perceived stress, life events, dysfunctional attitudes, and depression in adolescent psychiatric inpatients. J. Psychopathol. Behav. Assess. 17, 81-95. doi:10.1007/BF02229205 
Miller. G., Chapman, J., 2001. Misunderstanding analysis of covariance. J. Abnorm. Psychol., 110, 40-48. http://www.ncbi.nlm.nih.gov/pubmed/11261398

Mynard, H., Joseph, S., 2000. Development of the multidimensional peer-victimization scale. Aggressive Behav. 26, 169-178. doi:10.1002/(SICI)1098-2337(2000)26:2<169::AID$\mathrm{AB} 3>3.0 . \mathrm{CO} ; 2-\mathrm{A}$

Nelson, E.E., Jarcho, J.M., Guyer, A.E., 2016. Social re-orientation and brain development: An expanded and updated view. Dev. Cogn. Neurosci. 17, 118-127. doi:10.1016/j.dcn.2015.12.008

Noone, D., Ames, C., Hassanali, N., Browning, S., Bracegirdle, K., Corrigall, R., Laurens, K.R., Hirsch, C.R., Kuipers, E., Maddox, L., Fowler, D., Jolley, S., 2015. A preliminary investigation of schematic beliefs and unusual experiences in children. Eur. Psychiatry 30, 569-575. doi:10.1016/j.eurpsy.2014.12.006

Palmier-Claus, J.E., Dunn, G., Lewis, S.W., 2012. Emotional and symptomatic reactivity to stress in individuals at ultra-high risk of developing psychosis. Psychol. Med. 42, 10031012. doi:10.1017/S0033291711001929

Peralta, V., Cuesta, M.J., 1994. Psychometric properties of the positive and negative syndrome scale (PANSS) in schizophrenia. Psychiatry Res. 53, 31-40.

Perneger, T., 1998. What's wrong with Bonferroni adjustments? BMJ, 316, 1236-1238. Available at: http://www.ncbi.nlm.nih.gov/pmc/articles/PMC1112991/

Pestle, S.L., Chorpita, B.F., Schiffman, J., 2008. Psychometric properties of the Penn State Worry Questionnaire for children in a large clinical sample. J. Clin. Child Adolesc. Psychol. 37, 465-71. doi:10.1080/15374410801955896

Pisano, S., Catone, G., Pascotto, A., Iuliano, R., Tiano, C., Milone, A., Masi, G., Gritti, A., 2015. Paranoid thoughts in adolescents with social anxiety disorder. Child Psychiatry Hum. Dev. 47, 1-7. doi:10.1007/s10578-015-0612-5

Raes, F., Van Gucht, D., 2009. Paranoia and instability of self-esteem in adolescents. Pers. Individ. Dif. 47, 928-932. doi:10.1016/j.paid.2009.07.017

Ronald, A., Sieradzka, D., Cardno, A.G., Haworth, C.M.A., McGuire, P., Freeman, D., 2014. Characterization of psychotic experiences in adolescence using the specific psychotic experiences questionnaire: Findings from a study of 5000 16-Year-Old Twins. Schizophr. Bull. 40, 868-877. doi:10.1093/schbul/sbt106

Rosenberg, M., 1965. Society and the adolescent self-image. Princeton University Press, Princeton NJ. 
Ruffell, T., Azis, M., Hassanali, N., Ames, C., Browning, S., Bracegirdle, K., Corrigall, R., Laurens, K.R., Hirsch, C., Kuipers, E., Maddox, L., Jolley, S., 2016. Variation in psychosocial influences according to the dimensions and content of children's unusual experiences: potential routes for the development of targeted interventions. Eur. Child Adolesc. Psychiatry 25, 311-319. doi:10.1007/s00787-015-0739-8

Sebastian, C., Viding, E., Williams, K.D., Blakemore, S.J., 2010. Social brain development and the affective consequences of ostracism in adolescence. Brain Cogn. 72, 134-145. doi:10.1016/j.bandc.2009.06.008

Shakoor, S., McGuire, P., Cardno, A.G., Freeman, D., Plomin, R., Ronald, A., 2015. A shared genetic propensity underlies experiences of bullying victimization in late childhood and self-rated paranoid thinking in adolescence. Schizophr. Bull. 41, 754-763. doi:10.1093/schbul/sbu142

Shi, Y., Luo, Y., Yang, Z., Liu, Y., Cai, H., 2014. The development and validation of the social network sites (SNSs) usage questionnaire. Lect. Notes Comput. Sci. 8531 LNCS, 113124. doi:10.1007/978-3-319-07632-4_11

Sinclair, S.J., Blais, M., Gansler, D., Sandberg, E., Bistis, K., LoCicero, A., 2010. Psychometric properties of the Rosenberg Self-Esteem Scale: Overall and across demographic groups living within the United States. Eval. Health Prof. 33, 56-80. doi:10.1177/0163278709356187

Taylor, M.J., Gregory, A.M., Freeman, D., Ronald, A., 2015. Do sleep disturbances and psychotic-like experiences in adolescence share genetic and environmental influences ? J. Abnorm. Psychol. 124, May 4 [Epub ahead of print]. doi:10.1037/abn0000057 Volk, A., Craig, W., Boyce, W., King, M., 2006. Adolescent risk correlates of bullying and different types of victimization. Int. J. Adolesc. Med. Health 18, 575-586. doi:10.1515/IJAMH.2006.18.4.575

Wigman, J.T.W., Vollebergh, W.A.M., Raaijmakers, Q.A.W., Iedema, J., Van Dorsselaer, S., Ormel, J., Verhulst, F.C., Van Os, J., 2011. The structure of the extended psychosis phenotype in early adolescence - A cross-sample replication. Schizophr. Bull. 37, 850860. doi:10.1093/schbul/sbp154

Wong, K.K., Freeman, D., Hughes, C., 2014. Suspicious young minds: Paranoia and mistrust in 8- To 14-year-olds in the UK and Hong Kong. Br. J. Psychiatry 205, 221-229. doi:10.1192/bjp.bp.113.135467 
Zavos, H.M.S., 2014. Consistent etiology of severe, frequent psychotic experiences and milder, less frequent manifestations: A twin study of specific psychotic experiences in adolescence. JAMA Psychiatry 71, 1049-1057. doi:10.1001/jamapsychiatry.2014.994. 


\section{Tables}

Table 1.

Participant characteristics

\begin{tabular}{lcc}
\hline Variable & $\mathbf{n}$ & $\mathbf{\%}$ \\
\hline Female & 28 & $82 \%$ \\
Male & 6 & $18 \%$ \\
Ethnicity & 30 & \\
White British & 2 & $88 \%$ \\
White Other & 2 & $6 \%$ \\
Mixed ethnicity & & $6 \%$ \\
Primary presenting problem & 20 & \\
Anxiety and/or depression & 7 & $59 \%$ \\
Post-traumatic stress & 4 & $21 \%$ \\
Psychosis & 2 & $12 \%$ \\
Bipolar disorder & 1 & $6 \%$ \\
Emerging personality disorder & $\mathbf{1 6}$ & $3 \%$ \\
Receiving Medication & 11 & $\mathbf{4 7 \%}$ \\
Antidepressant & 6 & $32 \%$ \\
Antipsychotic & 4 & $18 \%$ \\
Hypnotic & 1 & $12 \%$ \\
Mood stabilizer & 1 & $3 \%$ \\
Anti-anxiety & $\mathbf{1 9}$ & $3 \%$ \\
Receiving therapy & 12 & $\mathbf{5 6 \%}$ \\
CBT & 4 & $35 \%$ \\
Psychodynamic therapies & 2 & $12 \%$ \\
DBT & 1 & $6 \%$ \\
Family therapy & & $3 \%$ \\
\hline
\end{tabular}


Table 2.

Descriptive statistics

\begin{tabular}{lll}
\hline Variables & Mean & SD \\
\hline Paranoia (GPTS) - Baseline & 51.5 & 12.4 \\
Paranoia (GPTS) - Follow up & 45.8 & 17.1 \\
Depression & 18.7 & 5.87 \\
Anxiety total & 60.3 & 15.3 \\
Worry & 32.0 & 5.05 \\
Negative core beliefs & 12.9 & 5.26 \\
Self-esteem & 19.4 & 6.15 \\
Academic competence & 10.5 & 3.46 \\
Social competence & 9.74 & 4.20 \\
Physical appearance & 8.03 & 4.25 \\
Perceptual anomalies & 20.0 & 10.9 \\
JTC - Number of beads & 7.88 & 2.90 \\
Perceived Stress (PSS) ${ }^{\text {a }}$ & 38.2 & 6.10 \\
Social media: Usage & 53.4 & 11.5 \\
Social media: Negative affect ${ }^{\text {b }}$ & 14.4 & 5.57 \\
Cyber victimization & 36.1 & 11.6 \\
Peer bullying & 16.0 & 8.60 \\
\hline Notes: ${ }^{\text {a. }}$ PSS $n=33$ due to one participant being excluded due to missing data exceeding 20\%. ${ }^{\text {b. SNS negative }}$ \\
affective $n=31$ as 3 participants did not use social media. &
\end{tabular}


Table 3.

Bivariate and partial correlations for variables and GPTS at baseline and follow up.

\begin{tabular}{|c|c|c|c|c|c|c|}
\hline \multicolumn{7}{|c|}{ Correlations with paranoia } \\
\hline & \multicolumn{2}{|c|}{ Baseline } & \multicolumn{2}{|c|}{ Follow up } & \multicolumn{2}{|c|}{$\begin{array}{c}\text { Follow up } \\
\text { (baseline adjusted }^{\text {a) }} \text { ) }\end{array}$} \\
\hline & $r$ & $p$ & $r$ & $p$ & $r$ & $p$ \\
\hline$\overline{\text { Depression }}$ & 0.56 & $<0.001$ & 0.47 & $<0.008$ & 0.12 & $<0.527$ \\
\hline Anxiety total & 0.56 & $<0.001$ & 0.52 & $<0.002$ & 0.23 & $<0.207$ \\
\hline Worry & 0.34 & $<0.048$ & 0.47 & $<0.006$ & 0.34 & $<0.057$ \\
\hline Negative core beliefs & 0.51 & $<0.002$ & 0.57 & $<0.001$ & 0.34 & $<0.059$ \\
\hline Self esteem & -0.46 & $<0.007$ & -0.43 & $<0.012$ & -0.18 & $<0.326$ \\
\hline Academic competence & -0.17 & $<0.328$ & -0.20 & $<0.277$ & -0.11 & $<0.567$ \\
\hline Social competence ${ }^{\mathbf{b}}$ & 0.09 & $<0.454$ & 0.12 & $<0.364$ & 0.11 & $<0.549$ \\
\hline Perceptual anomalies & 0.54 & $<0.001$ & 0.39 & $<0.026$ & 0.01 & $<0.954$ \\
\hline Insomnia & 0.37 & $<0.033$ & 0.51 & $<0.003$ & 0.38 & $<0.035$ \\
\hline Perceived stress & 0.51 & $<0.003$ & 0.65 & $<0.001$ & 0.47 & $<0.007$ \\
\hline Social media use & 0.16 & $<0.362$ & 0.26 & $<0.143$ & 0.21 & $<0.250$ \\
\hline Social media affect & 0.36 & $<0.073$ & 0.53 & $<0.002$ & 0.45 & $<0.014$ \\
\hline Cyber victimization $^{\mathbf{c}}$ & 0.29 & $<0.126$ & 0.39 & $<0.025$ & 0.31 & $<0.088$ \\
\hline Peer bullying & 0.56 & $<0.001$ & 0.59 & $<0.001$ & 0.33 & $<0.063$ \\
\hline
\end{tabular}

